\title{
On the choice of stress-strain variables for unsaturated soils and its effect on plastic flow
}

Domenico Gallipolia, Peter Grass ${ }^{b}$, Simon Wheeler ${ }^{c}$ and Antonio Gens ${ }^{d}$

a Professor, Université de Pau et des Pays de l'Adour, Laboratoire SIAME, Fédération IPRA, 64600, Anglet, France - domenico.gallipoli@univ-pau.fr

b Senior Lecturer, University of Glasgow, School of Engineering, G12 8LT, Glasgow, United Kingdom - peter.grass|@glasgow.ac.uk

c Professor, University of Glasgow, School of Engineering, G12 8LT, Glasgow, United Kingdom - simon.wheeler@glasgow.ac.uk

d Professor, Universitat Politècnica de Catalunya, Departament d'Enginyeria Civil i Ambiental, 08034, Barcelona, Spain - antonio.gens@upc.edu

DATE OF SUBMISSION: 20/12/2017

NUMBER OF WORDS: 4139

NUMBER OF FIGURES: 2

CORRESPONDING AUTHOR: Prof Domenico Gallipoli

Université de Pau et des Pays de l'Adour

Laboratoire SIAME, Fédération IPRA

Bâtiment ISABTP

Allée du Parc Montaury

64600 Anglet, France

Tel: +33559574280

e-mail: domenico.gallipoli@univ-pau.fr 
ABSTRACT: The net stress plus suction and the average skeleton stress plus modified suction are two alternative sets of energetically consistent stress variables for modelling the hydro-mechanical behaviour of unsaturated soils. When used in conjunction with their work-conjugate strains, both sets of stress variables correctly calculate the firstorder term of the hydro-mechanical work input into a soil element subjected to infinitesimal changes of deformation and water content. They therefore also correctly calculate the increment of internal energy along a given stress-strain path, that is the integral of the first-order term of the infinitesimal work input.

This paper shows, however, that the above two sets of stress variables lead to different expressions of the second-order term of the hydro-mechanical work input. The above sets are therefore no longer equivalent with respect to other aspects of material
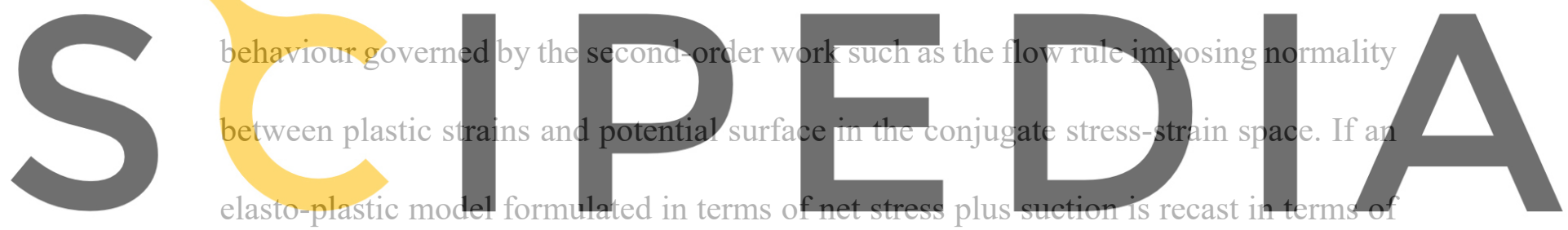

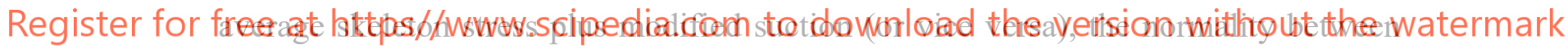

plastic strain vectors and potential surface is lost. To restore normality in both stress spaces, it is necessary to impose specific forms of elastic and plastic behaviour.

KEYWORDS: unsaturated soils; effective stress; suction, work input; plastic flow; elasto-plasticity. 


\section{INTRODUCTION}

Since Alonso et al. [1] published the first elasto-plastic model for unsaturated soils, many alternative constitutive formulations have been proposed with the most recent ones coupling mechanical and hydraulic behaviour in a single analytical framework. An important aspect of these models is the choice of stress-strain variables. A large variety of possibilities exists in the literature as discussed, for example, by Gens [2] and D'Onza et al. [3]. Some stress-strain variables have been chosen because of experimental convenience, i.e. variables that are easier to measure or control during laboratory testing [4]. Other stress-strain variables have instead been chosen because of theoretical rigour, i.e. variables originating from a thermodynamic analysis and/or a physical interpretation of microscopic behaviour, as in the works of Houlsby [5],
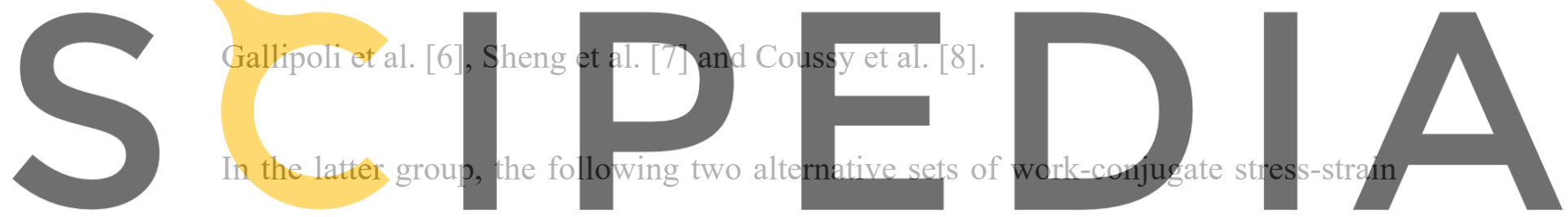

variables are commonly used because they both allow calculation of the internal energy Register for free at https//www.scipedia.com to download the version without the watermark change along a given stress-strain path:

(1) The net stress (mechanical stress) plus suction (hydraulic stress) and the Cauchy strain (mechanical strain) plus water ratio strain (hydraulic strain) (Vaunat et al. [9] provide an example of a constitutive model using this set of variables);

(2) The average skeleton stress (mechanical stress) plus modified suction (hydraulic stress) and the Cauchy strain (mechanical strain) plus water saturation strain (hydraulic strain) (Lloret-Cabot et al. [10] ; [11] provide an example of a constitutive model using this set of variables). 
The above two sets of stress-strain variables correctly calculate the first-order term of the infinitesimal hydro-mechanical work input into an unsaturated soil element. Therefore, they also correctly calculate the change of internal energy along a given stress-strain path, which coincides with the integral of the first-order term of the work input under adiabatic conditions.

However, as shown in this paper, the above two sets of variables lead to different expressions of the second-order term of the infinitesimal work input. This has implications for those aspects of material behaviour that are governed by the secondorder work such as the flow rule in elasto-plastic models. In particular, the normality between plastic strain vectors and potential function is lost when both these quantities are mapped from one stress space to the other. This violates the very definition of plastic
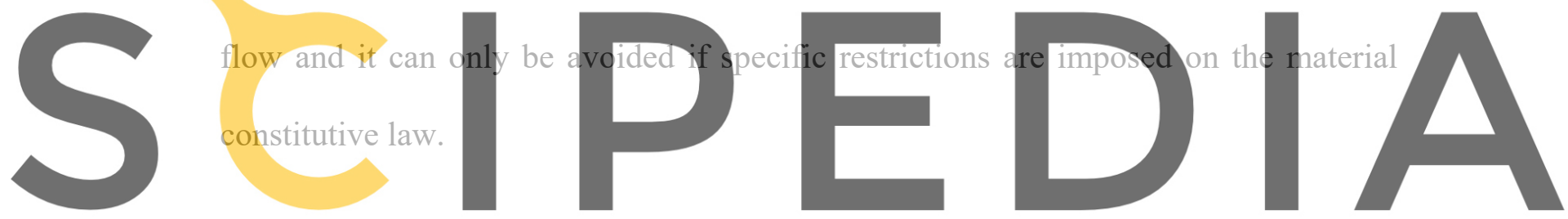

2. WORK INPUT PER UNIT VOLUME

Register for free at https//www.scipedia.com to download the version without the watermark

For a single-phase material, the infinitesimal mechanical work input limited to the second order term is expressed as:

$$
\mathrm{d} W=\mathrm{d} W^{(1)}+\mathrm{d} W^{(2)}
$$

where the first- and second-order terms are respectively defined as:

$$
\mathrm{d} W^{(1)}=\sigma_{i j} \mathrm{~d} \epsilon_{i j}
$$

and 


$$
\mathrm{d} W^{(2)}=\frac{1}{2} \mathrm{~d} \sigma_{i j} \mathrm{~d} \epsilon_{i j}
$$

with $\sigma_{i j}$ and $\epsilon_{i j}$ being the stress and strain variables, respectively.

The increment of internal energy of a material subjected to a given stress-strain path is calculated by integration of the first-order term $\mathrm{d} W^{(1)}$ as the second-order term $\mathrm{d} W^{(2)}$ is comparatively negligible. The second-order term of the work input may however be important for reasons other than calculating the change of internal energy. For example,

Drucker [12] postulated that the positiveness of the second-order work is a sufficient condition to ensure the stable response of a material subjected to controlled loading [13]; [14]:
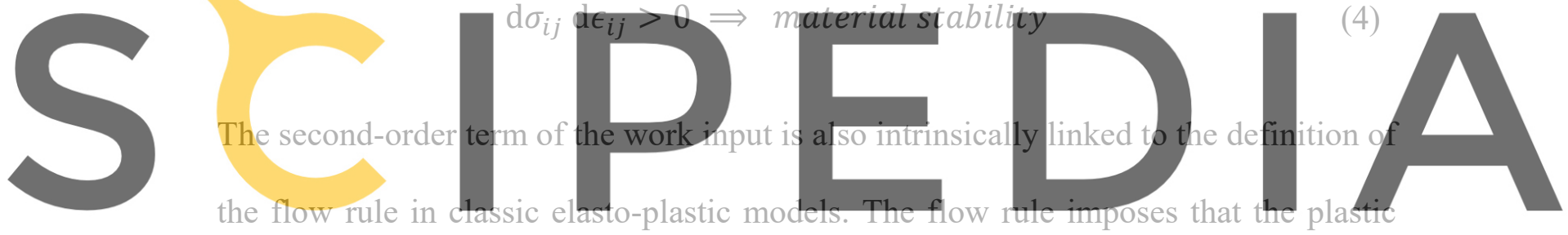

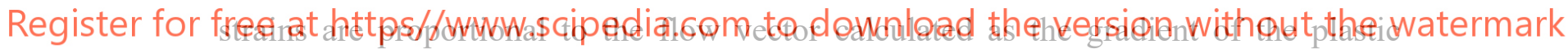

potential function defined in the conjugate stress space. Plastic strains are therefore normal to equipotential surfaces, which in turn implies that the second-order plastic work must be zero for any stress increment tangent to these surfaces. This aspect is further investigated in the present paper with specific reference to three-phase porous materials like unsaturated soils. The hydro-mechanical behaviour of these materials can be described by two alternative sets of energetically consistent stress variables, i.e. the net stress plus suction and the average skeleton stress plus modified suction, and their respective conjugate strains, i.e. the Cauchy strain plus water ratio strain and the Cauchy strain plus water saturation strain. In particular, the present paper explores whether the normality between equipotential surfaces and plastic strain vectors is 
preserved when both these geometrical quantities are mapped from one stress-strain space to the other. The mapping relationships between the two spaces are simply derived from the definitions of the stress and strain variables.

The paper starts by comparing the expressions of the first-order terms of the hydromechanical work input in the two stress-strain spaces. This does not bring any new knowledge but facilitates the subsequent analysis of the second-order terms, which provides the basis for the further study of plastic flow.

\section{First-order work input in an unsaturated soil}

The definitions of work input given by equations (2) and (3) apply to single-phase materials. In the case of three-phase materials such as unsaturated soils, these
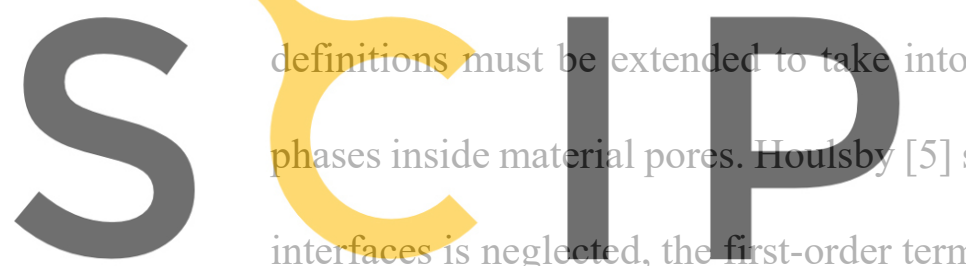

interfaces is neglected, the first-order term
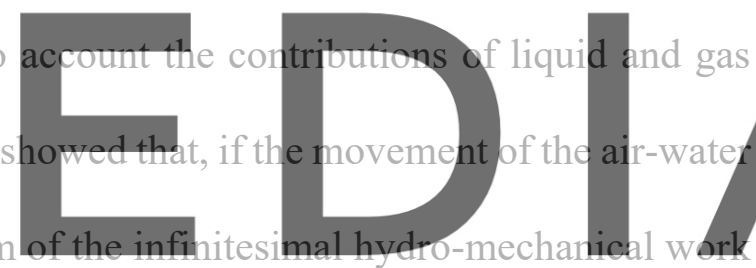

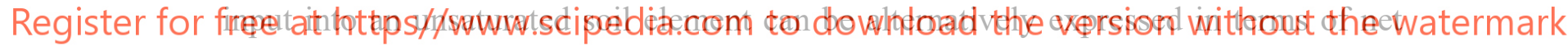

stress plus suction or average skeleton stress plus modified suction as:

$$
\begin{gathered}
\mathrm{d} W^{(1)}\langle\text { Form } 1\rangle=\sigma_{i j}^{\text {net }} d \epsilon_{i j}+s \mathrm{~d} \epsilon_{w} \\
d W^{(1)}\langle\text { Form } 2\rangle=\sigma_{i j}^{\prime} \mathrm{d} \epsilon_{i j}+s^{\prime} \mathrm{d}\left(-S_{r}\right)
\end{gathered}
$$

where:

1. $\sigma_{i j}^{\text {net }}$ and $s$ are the net stress (tensor) and suction (scalar) respectively defined as: 


$$
\begin{gathered}
\sigma_{i j}^{n e t}=\sigma_{i j}-u_{a} \delta_{i j} \\
s=u_{a}-u_{w}
\end{gathered}
$$

with $\sigma_{i j}$ being the total stress (tensor), $u_{a}$ the pore air pressure (scalar), $u_{w}$ the pore water pressure (scalar) and $\delta_{i j}$ the Kronecker's delta.

2. $\sigma_{i j}^{\prime}$ and $s^{\prime}$ are the average skeleton stress (tensor) and the modified suction (scalar) defined as :

$$
\sigma_{i j}^{\prime}=\sigma_{i j}-\left(S_{r} u_{w}+\left(1-S_{r}\right) u_{a}\right) \delta_{i j}=\sigma_{i j}^{n e t}+S_{r} S \delta_{i j}
$$

$$
s^{\prime}=n s
$$
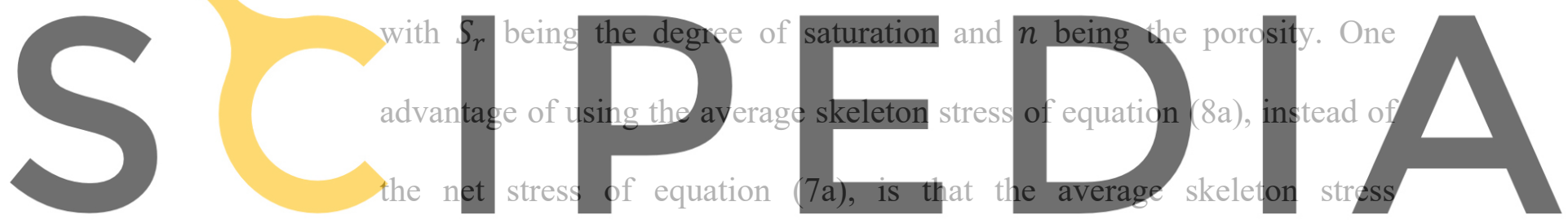

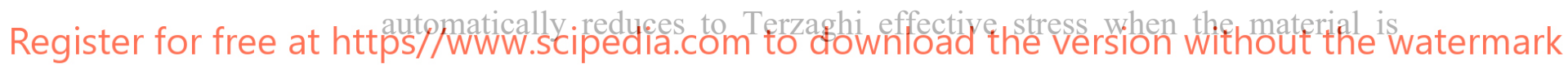

saturated by water $\left(S_{r}=1\right)$ or by $\operatorname{air}\left(S_{r}=0\right)$.

3. $\epsilon_{i j}$ is the Cauchy strain (tensor), which is conjugate of the net stress $\sigma_{i j}^{\text {net }}$ and of the average skeleton stress $\sigma_{i j}^{\prime}$.

4. $\epsilon_{w}$ is the water ratio strain (scalar), which is conjugate of suction $s$. The infinitesimal change of water ratio strain is defined as:

$$
\mathrm{d} \epsilon_{w}=-\frac{\mathrm{d} e_{w}}{1+e}
$$

where $e$ is the void ratio and $e_{w}$ is the water ratio defined as the volume of water per unit volume of solids. A decrease of water ratio produces an 
increase of water ratio strain due to the minus sign in equation (9), which is consistent with the soil mechanics convention of compression positive volumetric strains.

5. $-S_{r}$ is the water saturation strain (scalar), which is simply the negative of the degree of saturation $S_{r}=\frac{e_{w}}{e}$, and is conjugate of the modified suction $s^{\prime}$. The infinitesimal change of the water saturation strain is therefore expressed as:

$$
\mathrm{d}\left(-S_{r}\right)=\mathrm{d}\left(-\frac{e_{w}}{e}\right)=\frac{e_{w} \mathrm{~d} e-e \mathrm{~d} e_{w}}{e^{2}}=\frac{S_{r} \mathrm{~d} e-\mathrm{d} e_{w}}{e}
$$
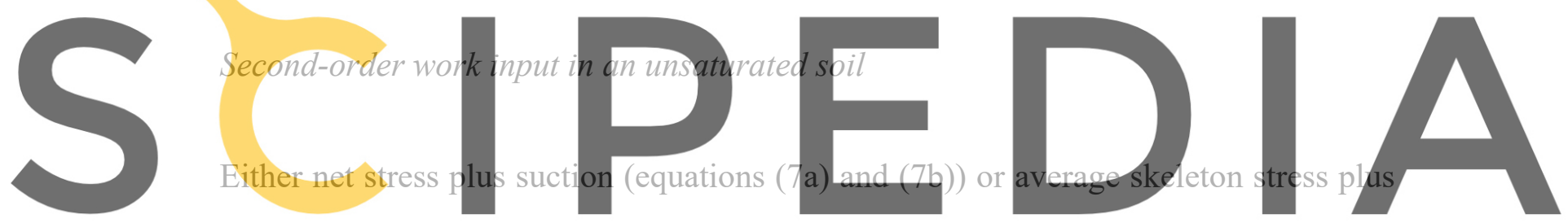

modified suction (equations (8a) and (8b)) provide an adequate set of stress variables

Register for free at https//www.scipedia.com to download the version without the watermark

for calculating the first-order term of the hydro-mechanical work input when used in

conjunction with their respective conjugate strains. The same is however not true for the second-order term of the hydro-mechanical work input, whose value changes depending on the chosen set of stress variables. To show this, the second-order term of the work input is here defined in terms of both net stress plus suction and average skeleton stress plus modified suction according to the following two alternative forms:

$$
\begin{gathered}
\mathrm{d} W^{(2)}\langle\text { Form } 1\rangle=\frac{1}{2}\left(\mathrm{~d} \sigma_{i j}^{n e t} \mathrm{~d} \epsilon_{i j}+\mathrm{d} s \mathrm{~d} \epsilon_{w}\right) \\
\mathrm{d} W^{(2)}\langle\text { Form } 2\rangle=\frac{1}{2}\left(\mathrm{~d} \sigma_{i j}^{\prime} \mathrm{d} \epsilon_{i j}+\mathrm{d} s^{\prime} \mathrm{d}\left(-S_{r}\right)\right)
\end{gathered}
$$


To compare the above two forms we map the second one, i.e. equation (12), from the space of average skeleton stress plus modified suction to the space of net stress plus suction. For this, the relationships between stress increments in the two spaces are first obtained through differentiation of the average skeleton stress and modified suction expressions of equations ( $8 a$ ) and (8b), respectively. This leads to the expression of the increments $\mathrm{d} \sigma_{i j}^{\prime}$ and $\mathrm{d} s^{\prime}$ in terms of the increments $\mathrm{d} \sigma_{i j}^{n e t}$ and $\mathrm{d} s$ as:

$$
\begin{gathered}
\mathrm{d} \sigma_{i j}^{\prime}=\mathrm{d} \sigma_{i j}^{n e t}+S_{r} \mathrm{~d} s \delta_{i j}+s \mathrm{~d} S_{r} \delta_{i j} \\
\mathrm{~d} s^{\prime}=n \mathrm{~d} s+s \mathrm{~d} n
\end{gathered}
$$

which are then substituted into equation (12) to give:
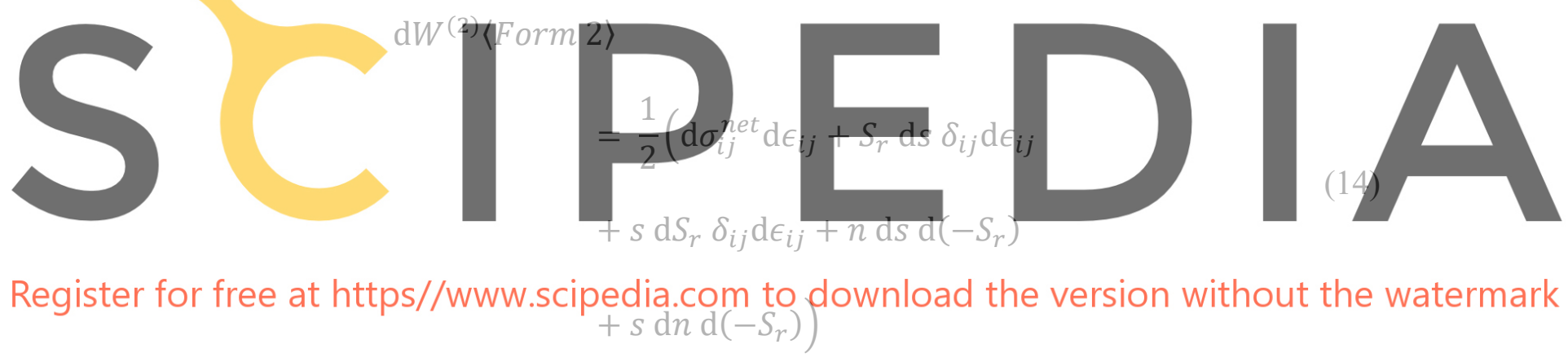

Next, the relationship between the increments of water saturation strain $\mathrm{d}\left(-S_{r}\right)$ and water ratio strain $\mathrm{d} \epsilon_{w}$ is obtained by substituting into equation (10) the definitions of porosity $n=\frac{e}{1+e}$, incremental water ratio strain $\mathrm{d} \epsilon_{w}=-\frac{\mathrm{d} e_{w}}{1+e}$ and incremental volumetric strain $\delta_{i j} \mathrm{~d} \epsilon_{i j}=-\frac{\mathrm{d} e}{1+e}($ compression positive):

$$
\mathrm{d}\left(-S_{r}\right)=\frac{\mathrm{d} \epsilon_{w}-S_{r} \delta_{i j} \mathrm{~d} \epsilon_{i j}}{n}
$$


By substituting equation (15) into equation (14) and noting that $\mathrm{d} n=(n-1) \delta_{i j} \mathrm{~d} \epsilon_{i j}$, the second form of equation (12) is finally recast in terms of net stress plus suction and corresponding conjugate strains as:

$$
\begin{aligned}
& \mathrm{d} W^{(2)}\langle\text { Form 2 }\rangle \\
& \quad=\frac{1}{2}\left(\mathrm{~d} \sigma_{i j}^{n e t} \mathrm{~d} \epsilon_{i j}+\mathrm{d} s \mathrm{~d} \epsilon_{w}\right)-\frac{1}{2} \frac{s(2-n)}{n}\left(\delta_{i j} \mathrm{~d} \epsilon_{i j} \mathrm{~d} \epsilon_{w}-S_{r}\left(\delta_{i j} \mathrm{~d} \epsilon_{i j}\right)^{2}\right)
\end{aligned}
$$

Comparison of equations (11) and (16) shows a difference, which implies that one of the two forms must be incorrect. Equation (11) coincides with the expression of the second-order work derived by Buscarnera and di Prisco [15] via an energy balance approach similar to that followed by Houlsby [5] for deriving the expression of the firstorder work. This suggests that equation (11) is correct while equation (12) is incorrect,
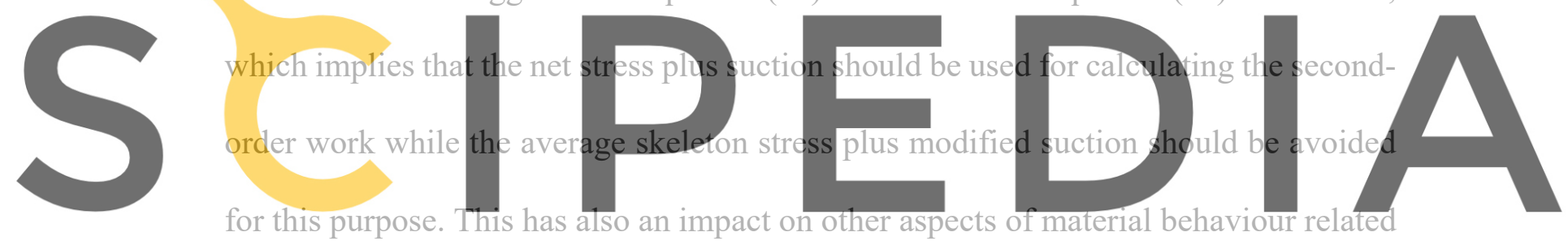

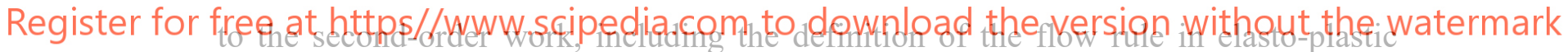

modeis as it will be discussed in the next section. It is aiso worth emphasizing that the above result has no implications for material energy and the two sets of variables are both energetically consistent. This is because, as shown by Houlsby [5], both sets of variables correctly calculate the first-order work whose integration gives the change of internal energy along a generic stress-strain path.

The difference $E^{(2)}$ between the two forms of equations (11) and (16) is:

$$
E^{(2)}=-\frac{1}{2} \frac{s(2-n)}{n}\left(\delta_{i j} \mathrm{~d} \epsilon_{i j} \mathrm{~d} \epsilon_{w}-S_{r}\left(\delta_{i j} \mathrm{~d} \epsilon_{i j}\right)^{2}\right)
$$


which can alternatively be expressed in terms of $\mathrm{d}\left(-S_{r}\right)$ instead of $\mathrm{d} \epsilon_{w}$ by recalling equations (15) :

$$
E^{(2)}=-\frac{1}{2} s(2-n) \delta_{i j} \mathrm{~d} \epsilon_{i j} \mathrm{~d}\left(-S_{r}\right)
$$

Given that suction $s$ is generally positive and porosity $n$ is bound between zero and one, this difference only vanishes for strain paths where no change of either volumetric or saturation strain occurs, i.e. when either $\delta_{i j} \mathrm{~d} \epsilon_{i j}=0$ or $\mathrm{d}\left(-S_{r}\right)=0$.

\section{PLASTIC FLOW RULE}

The two second-order work forms calculated in the previous section are here exploited to investigate the normality between plastic flow vectors and equipotential surfaces when an elasto-plastic soil model formulated in the space of net stress plus suction is mapped to the space of average skeleton stress plus modified suction, or vice versa.

Let us first note that equation (15) can be separately written for the elastic and plastic strain components:

$$
\begin{aligned}
& \mathrm{d}\left(-S_{r}^{\mathrm{e}}\right)=\frac{d \epsilon_{w}^{\mathrm{e}}-S_{r} \delta_{i j} d \epsilon_{i j}^{\mathrm{e}}}{n} \\
& \mathrm{~d}\left(-S_{r}^{\mathrm{p}}\right)=\frac{\mathrm{d} \epsilon_{w}^{\mathrm{p}}-S_{r} \delta_{i j} \mathrm{~d} \epsilon_{i j}^{\mathrm{p}}}{n}
\end{aligned}
$$

where superscripts "e" and "p" indicate the elastic and plastic components of strains, respectively.

Consider now a material element at yielding which undergoes an infinitesimal change

of plastic strain represented as $\left(\mathrm{d} \epsilon_{i j}^{\mathrm{p}}, \mathrm{d} \epsilon_{w}^{\mathrm{p}}\right)$ in the conjugate strain space of net stress 
plus suction, and as $\left(\mathrm{d} \epsilon_{i j}^{\mathrm{p}}, \mathrm{d}\left(-S_{r}^{\mathrm{p}}\right)\right)$ in the conjugate strain space of average skeleton stress plus modified suction. Next, let us consider an arbitrary infinitesimal stress change, which is represented as $\left(\mathrm{d} \sigma_{i j}^{n e t}, \mathrm{~d} s\right)$ in the space of net stress plus suction, and as $\left(\mathrm{d} \sigma_{i j}^{\prime}, \mathrm{d} s^{\prime}\right)$ in the space of average skeleton stress plus modified suction. Note that the above are different representations (i.e. representations in distinct constitutive spaces) of the same infinitesimal increments of stresses and strains. It is therefore possible to change from one representation to the other by using the mapping relationships of equations (20), (13a) and (13b).

These different representations of stress and strain increments produce the following two different representations of the second-order plastic work:

$$
\begin{gathered}
\mathrm{d} W^{(2)}{ }^{\mathrm{p}}\langle\text { Form } 1\rangle=\frac{1}{2}\left(\mathrm{~d} \sigma_{i j}^{n e t} \mathrm{~d} \epsilon_{i j}^{\mathrm{p}}+\mathrm{d} s \mathrm{~d} \epsilon_{w}^{\mathrm{p}}\right) \\
\mathrm{d} W^{(2)}{ }^{\mathrm{p}}\langle\text { Form } 2\rangle=\frac{1}{2}\left(\mathrm{~d} \sigma_{i j}^{\prime} \mathrm{d} \epsilon_{i j}^{\mathrm{p}}+\mathrm{d} s^{\prime} \mathrm{d}\left(-S_{r}^{\mathrm{p}}\right)\right)
\end{gathered}
$$

For ease of comparison, the second form of equation (22) is mapped from the space of average skeleton stress plus modified suction to the space of net stress plus suction. To this end, the average skeleton stress and modified suction are first recast in terms of net stress and suction by using equations (13a) and (13b):

$$
\begin{aligned}
& \mathrm{d} W^{(2)}{ }^{\mathrm{p}}\langle\text { Form } 2\rangle \\
& =\frac{1}{2}\left(\left(\mathrm{~d} \sigma_{i j}^{n e t}+S_{r} \mathrm{~d} s \delta_{i j}+s \mathrm{~d} S_{r} \delta_{i j}\right) \mathrm{d} \epsilon_{i j}^{\mathrm{p}}\right. \\
& \left.+(s \mathrm{~d} n+n \mathrm{~d} s) \mathrm{d}\left(-S_{r}^{\mathrm{p}}\right)\right)
\end{aligned}
$$


Then, the increments of water saturation strain are recast in terms of the corresponding increments of water ratio strain by replacing $\mathrm{d}\left(-S_{r}^{\mathrm{p}}\right)$ with equation (20) and $\mathrm{d} S_{r}=$ $-\mathrm{d}\left(-S_{r}\right)$ with the opposite of equation (15) which, after noting that $\mathrm{d} n=$ $-(1-n) \delta_{i j} \mathrm{~d} \epsilon_{i j}$, yields:

$$
\begin{aligned}
& \mathrm{d} W^{(2)}{ }^{\mathrm{p}}\langle\text { Form } 2\rangle \\
& =\frac{1}{2}\left(\mathrm{~d} \sigma_{i j}^{n e t} \mathrm{~d} \epsilon_{i j}^{\mathrm{p}}+\mathrm{d} s \mathrm{~d} \epsilon_{w}^{\mathrm{p}}\right) \\
& -\frac{1}{2} \frac{s}{n}\left(\left(\mathrm{~d} \epsilon_{w}+(n-2) S_{r} \delta_{i j} \mathrm{~d} \epsilon_{i j}\right) \delta_{i j} \mathrm{~d} \epsilon_{i j}^{\mathrm{p}}\right. \\
& \left.+(1-n) \delta_{i j} \mathrm{~d} \epsilon_{i j} \mathrm{~d} \epsilon_{w}^{\mathrm{p}}\right)
\end{aligned}
$$

Comparison of equations (21) and (24) indicates again that the two forms of the secondorder plastic work do not coincide and that the difference $E^{(2)}{ }^{\mathrm{p}}$ is:

$$
\begin{gathered}
E^{(2)^{\mathrm{p}}=-\frac{1}{2} \frac{s}{n}}\left(\left(d \epsilon_{w}+(n-2) S_{r} \delta_{i j} d \epsilon_{i j}\right) \delta_{i j} d \epsilon_{i j}^{p}\right. \\
\left.+(1-n) \delta_{i j} d \epsilon_{i j} d \epsilon_{w}^{p}\right)
\end{gathered}
$$

Alternatively, by recalling equations (15) and (20), the difference $E^{(2)}{ }^{\mathrm{p}}$ can be expressed in terms of $\mathrm{d}\left(-S_{r}\right)$ and $\mathrm{d}\left(-S_{r}^{\mathrm{p}}\right)$ instead of $\mathrm{d} \epsilon_{w}$ and $\mathrm{d} \epsilon_{w}^{\mathrm{p}}$ as:

$$
\begin{aligned}
E^{(2)^{\mathrm{p}}}=-\frac{1}{2} s & \left(\mathrm{~d}\left(-S_{r}\right) \delta_{i j} \mathrm{~d} \epsilon_{i j}^{\mathrm{p}}\right. \\
& \left.+(1-n) \delta_{i j} \mathrm{~d} \epsilon_{i j} \mathrm{~d}\left(-S_{r}^{\mathrm{p}}\right)\right)
\end{aligned}
$$

The above result has some consequences on the definition of the plastic flow rule as explained in the following.

Normality of plastic flow vectors to plastic potential 
Let us consider an equipotential surface passing through a generic stress state at yielding. The mathematical expression of this surface can be mapped between the two spaces of average skeleton stress plus modified suction and net stress plus suction by using the stress definitions of equations (8a) and (8b).

The flow rule imposes that, in the space of net stress plus suction, the infinitesimal changes of conjugate plastic strains are proportional to a flow vector defined as the gradient of the potential function, $g$ as:

$$
\begin{gathered}
\mathrm{d} \epsilon_{i j}^{\mathrm{p}}=\lambda \frac{\partial g}{\partial \sigma_{i j}^{n e t}} \\
\mathrm{~d} \epsilon_{w}^{\mathrm{p}}=\lambda \frac{\partial g}{\partial s}
\end{gathered}
$$

where the constant of proportionality is given by plastic multiplier, $\lambda$.

Similarly, in the space of average skeleton stress plus modified suction, the flow rule imposes that the infinitesimal changes of conjugate plastic strains are proportional to a flow vector defined as the gradient of the potential function, $g^{\prime}$ as:

$$
\begin{gathered}
\mathrm{d} \epsilon_{i j}^{\mathrm{p}}=\lambda^{\prime} \frac{\partial g^{\prime}}{\partial \sigma_{i j}^{\prime}} \\
\mathrm{d}\left(-S_{r}^{\mathrm{p}}\right)=\lambda^{\prime} \frac{\partial g^{\prime}}{\partial s^{\prime}}
\end{gathered}
$$

where the constant of proportionality is given by the plastic multiplier, $\lambda^{\prime}$.

Taking into account equations (27) and (28), we rewrite the two forms of the secondorder plastic work given by equations (21) and (24) as: 


$$
\begin{array}{r}
\mathrm{d} W^{(2)}{ }^{\mathrm{p}}\langle\text { Form } 1\rangle=\frac{\lambda}{2}\left(\mathrm{~d} \sigma_{i j}^{\text {net }} \frac{\partial g}{\partial \sigma_{i j}^{\text {net }}}+\mathrm{d} s \frac{\partial g}{\partial s}\right) \\
\mathrm{d} W^{(2)}{ }^{\mathrm{p}}\langle\text { Form } 2\rangle \\
=\frac{\lambda}{2}\left(\mathrm{~d} \sigma_{i j}^{\text {net }} \frac{\partial g}{\partial \sigma_{i j}^{\text {net }}}+\mathrm{d} s \frac{\partial g}{\partial s}\right)+E^{(2)}{ }^{\mathrm{p}}
\end{array}
$$

Let us now assume an arbitrary stress increment tangent to the equipotential surface in the space of net stress plus suction. By definition of flow rule, the chosen stress increment vector $\left(\mathrm{d} \sigma_{i j}^{n e t}, \mathrm{~d} s\right)$ must be normal to the flow vector $\left(\frac{\partial g}{\partial \sigma_{i j}^{n e t}}, \frac{\partial g}{\partial s}\right)$, which means that $\mathrm{d} \sigma_{i j}^{n e t} \frac{\partial g}{\partial \sigma_{i j}^{n e t}}+\mathrm{d} s \frac{\partial g}{\partial s}=0$ and the second-order plastic work calculated by equation (31) vanishes:

$$
\mathrm{d} W^{(2)}{ }^{\mathrm{p}}\langle\text { Form } 1\rangle=0
$$

If the stress increments and plastic flow vectors were normal also in the space of average skeleton stress and modified suction, the second-order term of the plastic work calculated by equation (32) should be zero too. This is however not the case because, if $\mathrm{d} \sigma_{i j}^{n e t} \frac{\partial g}{\partial \sigma_{i j}^{n e t}}+\mathrm{d} s \frac{\partial g}{\partial s}=0$ is substituted in equation (32), we are left with:

$$
\mathrm{d} W^{(2)}{ }^{\mathrm{p}}\langle\text { Form } 2\rangle=E^{(2)^{\mathrm{p}}}
$$

In conclusion, the normality between the plastic flow vector and the equipotential surface is not preserved when these two geometrical quantities are mapped from the space of net stress plus suction to the space of average skeleton stress plus modified suction. Similarly, if the plastic flow vector is perpendicular to the equipotential surface in the space of average skeleton stress plus modified suction, this normality is lost when these two quantities are recast in the space of net stress plus suction. This is of course 
also true in the case of an associated flow rule, i.e. when potential and yield functions coincide, which means that associativeness is lost if the model is mapped between different stress spaces. This aspect is particularly relevant to finite element models because the assumption of an associated flow rule ensures the symmetry of the tangent stiffness matrix of the numerical model. Symmetric matrices can be easily inverted by means of efficient algorithms that cannot however be applied to asymmetric matrices. Mapping an associated model from one stress space to the other should therefore be weighted against potential disadvantages resulting from the loss of structural symmetry.

Normality is preserved in both stress spaces only if the right hand side of equation (34) is equal to zero, that is if $E^{(2)}{ }^{\mathrm{p}}=0$. This condition can be formulated in terms of $\mathrm{d} \epsilon_{w}$ and $\mathrm{d} \epsilon_{w}^{\mathrm{p}}$ or, alternatively, in terms of $\mathrm{d} S_{r}$ and $\mathrm{d} S_{r}^{\mathrm{p}}$ by imposing that either equation (25) or equation (26) is equal to zero. This gives the following two alternative expressions of the same restriction:

$$
\begin{gathered}
-\frac{1}{2} \frac{s}{n}\left(\left(\mathrm{~d} \epsilon_{w}+(n-2) S_{r} \delta_{i j} \mathrm{~d} \epsilon_{i j}\right) \delta_{i j} \mathrm{~d} \epsilon_{i j}^{\mathrm{p}}+(1-n) \delta_{i j} \mathrm{~d} \epsilon_{i j} \mathrm{~d} \epsilon_{w}^{\mathrm{p}}\right)=0 \\
-\frac{1}{2} s\left(\mathrm{~d}\left(-S_{r}\right) \delta_{i j} \mathrm{~d} \epsilon_{i j}^{\mathrm{p}}+(1-n) \delta_{i j} \mathrm{~d} \epsilon_{i j} \mathrm{~d}\left(-S_{r}^{\mathrm{p}}\right)\right)=0
\end{gathered}
$$

By using the plastic flow rule of equations (27) and (28), equation (35) is rewritten as:

$$
-\frac{\lambda}{2} \frac{s}{n}\left(\left(\mathrm{~d} \epsilon_{w}+(n-2) S_{r} \delta_{i j} \mathrm{~d} \epsilon_{i j}\right) \delta_{i j} \frac{\partial g}{\partial \sigma_{i j}^{n e t}}+(1-n) \delta_{i j} \mathrm{~d} \epsilon_{i j} \frac{\partial g}{\partial s}\right)=0
$$

Similarly, by using the plastic flow definitions of equations (29) and (30), equation (36) is rewritten as: 


$$
-\frac{\lambda^{\prime}}{2} s\left(\mathrm{~d}\left(-S_{r}\right) \delta_{i j} \frac{\partial g^{\prime}}{\partial \sigma_{i j}^{\prime}}+(1-n) \delta_{i j} \mathrm{~d} \epsilon_{i j} \frac{\partial g^{\prime}}{\partial s^{\prime}}\right)=0
$$

Given that suction and porosity are positive $(s \neq 0$ and $n \neq 0)$ and the plastic strain increment is non-null $\left(\lambda \neq 0\right.$ and $\left.\lambda^{\prime} \neq 0\right)$, equations (37) and (38) are only fulfilled if the following two conditions are satisfied:

$$
\begin{gathered}
\left(\mathrm{d} \epsilon_{w}+(n-2) S_{r} \delta_{i j} \mathrm{~d} \epsilon_{i j}\right) \delta_{i j} \frac{\partial g}{\partial \sigma_{i j}^{n e t}}+(1-n) \delta_{i j} \mathrm{~d} \epsilon_{i j} \frac{\partial g}{\partial s}=0 \\
\mathrm{~d}\left(-S_{r}\right) \delta_{i j} \frac{\partial g^{\prime}}{\partial \sigma_{i j}^{\prime}}+(1-n) \delta_{i j} \mathrm{~d} \epsilon_{i j} \frac{\partial g^{\prime}}{\partial s^{\prime}}=0
\end{gathered}
$$

Considering that $\delta_{i j} \frac{\partial g^{\prime}}{\partial \sigma_{i j}^{\prime}}=\frac{\partial g^{\prime}}{\partial p^{\prime}}$ (where $p^{\prime}$ is the mean average skeleton stress) and $\delta_{i j} \frac{\partial g}{\partial \sigma_{i j}^{n e t}}=\frac{\partial g}{\partial p^{n e t}}$ (where $p^{n e t}$ is the mean net stress) equations (39) and (40) can be further rewritten as:

$$
\begin{gathered}
\left(\mathrm{d} \epsilon_{w}+(n-2) S_{r} \delta_{i j} \mathrm{~d} \epsilon_{i j}\right) \frac{\partial g}{\partial p^{n e t}}+(1-n) \delta_{i j} \mathrm{~d} \epsilon_{i j} \frac{\partial g}{\partial s}=0 \\
\mathrm{~d}\left(-S_{r}\right) \frac{\partial g^{\prime}}{\partial p^{\prime}}+(1-n) \delta_{i j} \mathrm{~d} \epsilon_{i j} \frac{\partial g^{\prime}}{\partial s^{\prime}}=0
\end{gathered}
$$

Equation (41) imposes that $E^{(2)^{\mathrm{p}}}=0$ by enforcing a relationship between the plastic potential $g$ and the increments of Cauchy strain $\mathrm{d} \epsilon_{i j}$ and water ratio strain $\mathrm{d} \epsilon_{w}$ along the generic equipotential surface in the space of net stress plus suction. Similarly, equation (42) imposes that $E^{(2)^{\mathrm{p}}}=0$ by enforcing a relationship between the plastic potential $g^{\prime}$ and the increments of Cauchy strain $\mathrm{d} \epsilon_{i j}$ and water saturation strain $\mathrm{d}\left(-S_{r}\right)$ along the generic equipotential surface in the space of average skeleton stress 
plus modified suction. Note that equations (41) and (42) are different representations of the same restriction in two distinct stress spaces. Therefore, if one of them is verified, the other one is also automatically true.

The consequences of equations (41) and (42) are further investigated in the following with respect to the two cases of elastic and elasto-plastic stress increments along the generic equipotential surface.

\section{Case 1: elastic stress increment}

We first consider the case of an elastic increment along the generic equipotential surface. If the flow rule is associated, the stress state will move along the yield locus, which coincides with the equipotential surface (Figure 1a). Conversely, if the flow rule is not associated, the stress state will head inside the yield locus along the equipotential surface (Figure 1b).

Because the increments of water saturation strain $\mathrm{d}\left(-S_{r}\right)$ and Cauchy strain $\mathrm{d} \epsilon_{i j}$ coincide with their elastic components $\mathrm{d}\left(-S_{r}^{\mathrm{e}}\right)$ and $d \epsilon_{i j}^{\mathrm{e}}$, the two equations (41) and (42) can be rewritten as:

$$
\begin{gathered}
\left(\mathrm{d} \epsilon_{w}^{\mathrm{e}}+(n-2) S_{r} \delta_{i j} \mathrm{~d} \epsilon_{i j}^{\mathrm{e}}\right) \frac{\partial g}{\partial p^{n e t}}+(1-n) \delta_{i j} \mathrm{~d} \epsilon_{i j}^{\mathrm{e}} \frac{\partial g}{\partial s}=0 \\
\mathrm{~d}\left(-S_{r}^{\mathrm{e}}\right) \frac{\partial g^{\prime}}{\partial p^{\prime}}+(1-n) \delta_{i j} \mathrm{~d} \epsilon_{i j}^{\mathrm{e}} \frac{\partial g^{\prime}}{\partial s^{\prime}}=0
\end{gathered}
$$

and, by recalling that $\mathrm{d} n^{\mathrm{e}}=-(1-n) \delta_{i j} \mathrm{~d} \epsilon_{i j}^{\mathrm{e}}$ :

$$
\left(S_{r} \frac{n-2}{1-n} \mathrm{~d} n^{\mathrm{e}}-\mathrm{d} \epsilon_{w}^{\mathrm{e}}\right) \frac{\partial g}{\partial p^{n e t}}+\mathrm{d} n^{\mathrm{e}} \frac{\partial g}{\partial s}=0
$$




$$
\mathrm{d} S_{r}^{\mathrm{e}} \frac{\partial g^{\prime}}{\partial p^{\prime}}+\mathrm{d} n^{\mathrm{e}} \frac{\partial g^{\prime}}{\partial s^{\prime}}=0
$$

Equations (45) and (46) impose a restriction on the elastic law, and in particular on the admissible elastic changes of porosity, degree of saturation and water ratio strain along the generic equipotential surface. Once again, equations (45) and (46) are alternative forms of the same restriction but in different stress spaces. This restriction must be respected if the normality between plastic flow vectors and equipotential surfaces is to be preserved in both stress spaces. Of course, this restriction is automatically respected if elastic strains are neglected altogether.

\section{Case 2: elasto-plastic stress increment}

Next, we consider the case of an elasto-plastic increment along the generic equipotential surface. If the flow rule is associated and the model is non-strain-hardening, the stress state will move along the yield locus which coincides with the equipotential surface (Figure 1a). If the flow rule is instead not associated and the model is strain-hardening, then the stress state will head outside the yield locus along the equipotential surface (Figure 1b).

Note that, for the other two possibilities where the flow rule is associated and the model is strain-hardening or the flow rule is not associated and the model is non-strainhardening, only the previous case of an elastic increment can occur along the equipotential surface. Therefore, in these two instances, it is sufficient to satisfy only the elastic restriction imposed by equations (45) and (46) to preserve normality.

For an elasto-plastic increment, equations (41) and (42) can be rewritten by separating the elastic and plastic strains as: 


$$
\begin{aligned}
& \left(\mathrm{d} \epsilon_{w}^{\mathrm{e}}+(n-2) S_{r} \delta_{i j} \mathrm{~d} \epsilon_{i j}^{\mathrm{e}}\right) \frac{\partial g}{\partial p^{n e t}}+(1-n) \delta_{i j} \mathrm{~d} \epsilon_{i j}^{\mathrm{e}} \frac{\partial g}{\partial s} \\
& +\left(\mathrm{d} \epsilon_{w}^{\mathrm{p}}+(n-2) S_{r} \delta_{i j} \mathrm{~d} \epsilon_{i j}^{\mathrm{p}}\right) \frac{\partial g}{\partial p^{n e t}}+(1-n) \delta_{i j} \mathrm{~d} \epsilon_{i j}^{\mathrm{p}} \frac{\partial g}{\partial s}=0 \\
& \left(-\mathrm{d} S_{r}^{\mathrm{e}}\right) \frac{\partial g^{\prime}}{\partial p^{\prime}}+(1-n) \delta_{i j} \mathrm{~d} \epsilon_{i j}^{\mathrm{e}} \frac{\partial g^{\prime}}{\partial s^{\prime}}+\left(-\mathrm{d} S_{r}^{\mathrm{p}}\right) \frac{\partial g^{\prime}}{\partial p^{\prime}}+(1-n) \delta_{i j} \mathrm{~d} \epsilon_{i j}^{\mathrm{p}} \frac{\partial g^{\prime}}{\partial s^{\prime}}=0
\end{aligned}
$$

Here, we assume that the elastic restriction imposed by equations (45) and (46) are already satisfied so that equations (47) and (48) can be rewritten with reference only to plastic increments as:

$$
\begin{gathered}
\left(\mathrm{d} \epsilon_{w}^{\mathrm{p}}+(n-2) S_{r} \delta_{i j} \mathrm{~d} \epsilon_{i j}^{\mathrm{p}}\right) \frac{\partial g}{\partial p^{n e t}}+(1-n) \delta_{i j} \mathrm{~d} \epsilon_{i j}^{\mathrm{p}} \frac{\partial g}{\partial s}=0 \\
\left(-\mathrm{d} S_{r}^{\mathrm{p}}\right) \frac{\partial g^{\prime}}{\partial p^{\prime}}+(1-n) \delta_{i j} \mathrm{~d} \epsilon_{i j}^{\mathrm{p}} \frac{\partial g^{\prime}}{\partial s^{\prime}}=0
\end{gathered}
$$

By further taking into account the plastic flow rule of equations (27), (28), (29) and (30), we rewrite equations (49) and (50) as:

$$
\begin{gathered}
(2-n) \frac{\partial g}{\partial p^{n e t}}\left(\frac{\partial g}{\partial s}-S_{r} \frac{\partial g}{\partial p^{n e t}}\right)=0 \\
(2-n) \frac{\partial g^{\prime}}{\partial p^{\prime}} \frac{\partial g^{\prime}}{\partial s^{\prime}}=0
\end{gathered}
$$

Given that porosity $n$ is comprised between zero and one, equations (51) and (52) are fulfilled if the following conditions are respectively verified:

$$
\frac{\partial g}{\partial p^{n e t}}\left(\frac{\partial g}{\partial s}-S_{r} \frac{\partial g}{\partial p^{n e t}}\right)=0
$$




$$
\frac{\partial g^{\prime}}{\partial p^{\prime}} \frac{\partial g^{\prime}}{\partial s^{\prime}}=0
$$

Equations (53) and (54) are alternative representations, in different stress spaces, of the same restriction imposed this time on the plastic potential function. This restriction must be respected if the normality of the plastic flow rule is to be preserved in both stress spaces of net stress plus suction and average skeleton stress plus modified suction.

In the space of net stress plus suction, equation (53) requires that either the component $\frac{\partial g}{\partial p^{\text {net }}}$ of the plastic flow vector is zero or the ratio between the two components of the plastic flow vectors $\frac{\partial g}{\partial s} / \frac{\partial g}{\partial p^{\text {net }}}$ is equal to $S_{r}$. This requirement is met by a constitutive model where the equipotential surfaces in the $\left(p^{n e t}, s\right)$ plane are quadrilaterals with two sides parallel to the $p^{\text {net }}$ axis and the other two sides given by parallel lines with slope $-1 / S_{r}$.

Equivalently, in the space of average skeleton stress plus modified suction, equation (54) requires that at least one component of the plastic flow vector, that is either $\frac{\partial g^{\prime}}{\partial p^{\prime}}$ or $\frac{\partial g^{\prime}}{\partial s^{\prime}}$, is zero. This requirement is met by a constitutive model where the equipotential surfaces in the $\left(p^{\prime}, s^{\prime}\right)$ plane are rectangles with sides parallel to the $p^{\prime}$ and $s^{\prime}$ axes.

\section{CONCLUSIONS}

Multiple choices of stress-strain variables are possible to describe the deformation and water retention behaviour of unsaturated soils. Among these, the net stress plus suction and the average skeleton stress plus modified suction represent two alternative sets of energetically consistent stress variables. This means that, when used in conjunction 
with their conjugate strains, both sets of stresses correctly calculate the first-order work input and, by integration, the change of internal energy along a generic stress-strain path.

However, as shown in this paper, the net stress plus suction and the average skeleton stress plus modified suction calculate different expressions of the second-order work input. This difference only vanishes for strain paths that do not induce changes of either volumetric strain or degree of saturation. The correct expression of the second-order work is the one in terms of net stress plus suction as shown by Buscarnera and di Prisco [15]. This does not contradict the suitability of both sets of variables for calculating changes of internal energy because, as shown by Houlsby [5], both sets correctly calculate the first-order work whose integration along a stress-strain path gives the change of internal energy.

The paper has also shown that an elasto-plastic model formulated in terms of one set of stress-strain variables can be recast in the other set of stress-strain variables via standard mapping relationships between the two stress-strain spaces. However, if a model formulated in terms of net stress plus suction is recast in terms of average skeleton stress plus modified suction (or viceversa), the normality of the flow vectors to the equipotential surfaces may be lost, which of course violates the very definition of plastic potential. Moreover, for an associated flow rule, this loss of normality will result in a loss of symmetry of the stiffness matrix inside finite element models, which will produce a deterioration of algorithmic efficiency.

In order to preserve normality in both stress spaces, it is necessary to impose some restrictions on the constitutive law, i.e. specific forms of elastic behaviour and plastic potential must be assumed. 


\section{REFERENCES}

[1] Alonso E. E., Gens A. and Josa A. (1990). A constitutive model for partially $\begin{array}{llll}\text { saturated } & \text { soils. } & \text { Géotechnique }\end{array}$ http://dx.doi.org/10.1680/geot.1990.40.3.405

[2] Gens A. (2010). Soil-environment interactions in geotechnical engineering. Géotechnique 60(1): 3-74. https://doi.org/10.1680/geot.9.P.109

[3] D’Onza F., Gallipoli D., Wheeler S.J., Casini F., Vaunat J., Khalili N., Laloui L., Mancuso C., Masin D., Nuth M., Pereira J.M. and Vassallo R. (2011). Benchmark of constitutive models for unsaturated soils. Géotechnique 61(4): 285-304 http://dx.doi.org/10.1680/geot.2011.61.4.283

[4] Tarantino A., Gallipoli D., Augarde C., De Gennaro V., Gomez R., Laloui L., Mancuso C., McCloskey G., Munoz J., Pereira J.M., Peron H., Pisoni G., Romero E., Raveendiraraj A., Rojas J.C., Toll D., Tombolato S., Wheeler S.J. (2011). Benchmark of experimental techniques for measuring and controlling suction. Géotechnique 61(4): 303-312 - http://dx.doi.org/10.1680/geot.2011.61.4.303

[5] Houlsby G.T. (1997). The work input to an unsaturated granular material. Géotechnique 47(1): 193-196. http://dx.doi.org/10.1680/geot.1997.47.1.193

[6] Gallipoli D., Gens A., Sharma R. and Vaunat J. (2003). An elasto-plastic model for unsaturated soil including the effect of saturation degree on mechanical behaviour. $\quad$ Géotechnique 53(1): 123-135. http://dx.doi.org/10.1680/geot.53.1.123.37251 - Erratum. Géotechnique 53(9): 844. http://dx.doi.org/10.1680/geot.2003.53.9.844 
[7] Sheng D., Gens A., Fredlund D.G. and Sloan S.W. (2008). Unsaturated soils: From constitutive modelling to numerical algorithms. Computers and Geotechnics 35: 810-824 - http://dx.doi.org/10.1016/j.compgeo.2008.08.011

[8] Coussy O., Pereira J.M. and Vaunat J. (2010) Revisiting the thermodynamics of hardening plasticity for unsaturated soils. Computers and Geotechnics 37:207215. http://dx.doi.org/10.1016/j.compgeo.2009.09.003

[9] Vaunat J., Romero E. and Jommi C. (2000). An elastoplastic hydro-mechanical model for unsaturated soils. Experimental evidence and theoretical approaches in unsaturated soils (Eds. Tarantino A. and Mancuso C.), 121-138. Rotterdam: Balkema.

[10] Lloret-Cabot M., Sanchez M. and Wheeler S.J. (2013). Formulation of a threedimensional constitutive model for unsaturated soils incorporating mechanicalwater retention couplings. International Journal for Numerical and Analytical Methods in Geomechanics, 37:3008-3035.

[11] Lloret-Cabot M., Wheeler S.J. and Sanchez M. (2017). A unified mechanical and retention model for saturated and unsaturated soil behaviour. Acta Geotecnica, 12(1): 1-21.

[12] Drucker D.C. (1959). A definition of stable inelastic material. Journal of Applied Mechanics, 26:101-186. 
[13] Houlsby G.T. and Puzrin A.M. (2006). Principles of hyperplasticity: an approach to plasticity theory based on thermodynamic principles. Springer, ISBN: 9781846282393

[14] Darve F. and Laouafa F. (2000). Instabilities in granular materials and application to landslides. Mechanics of Cohesive-frictional Materials 5(8): 627-652. http://dx.doi.org/10.1002/1099-1484(200011)5:8<627::AID-

CFM109>3.0.CO;2-F

[15] Buscarnera G. and di Prisco C. (2012). Discussing the definition of the secondorder work for unsaturated soils. International Journal for Numerical and Analitycal Methods in Geomechanics 36(1): 36-49. http://dx.doi.org/10.1002/nag.991 


\section{FIGURES}

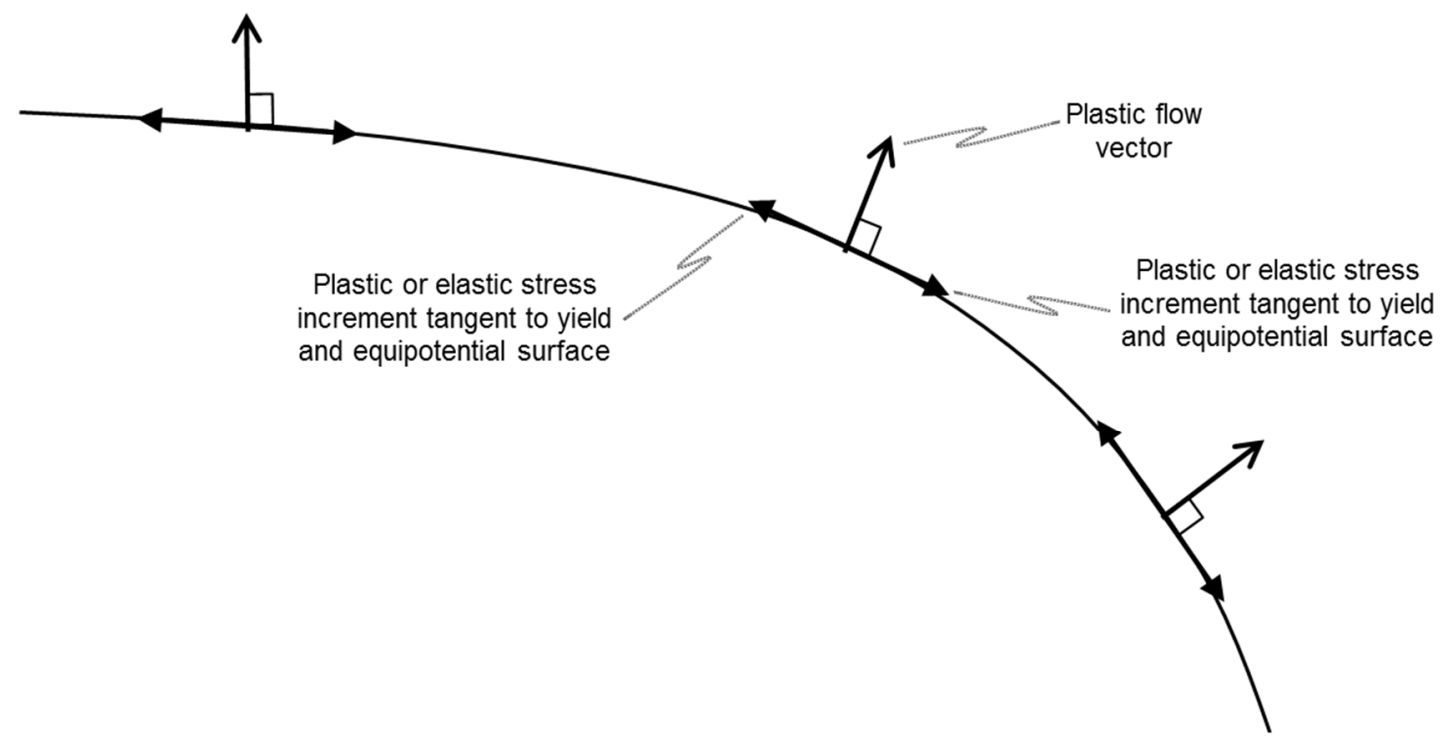

Yield and equipotential

a)

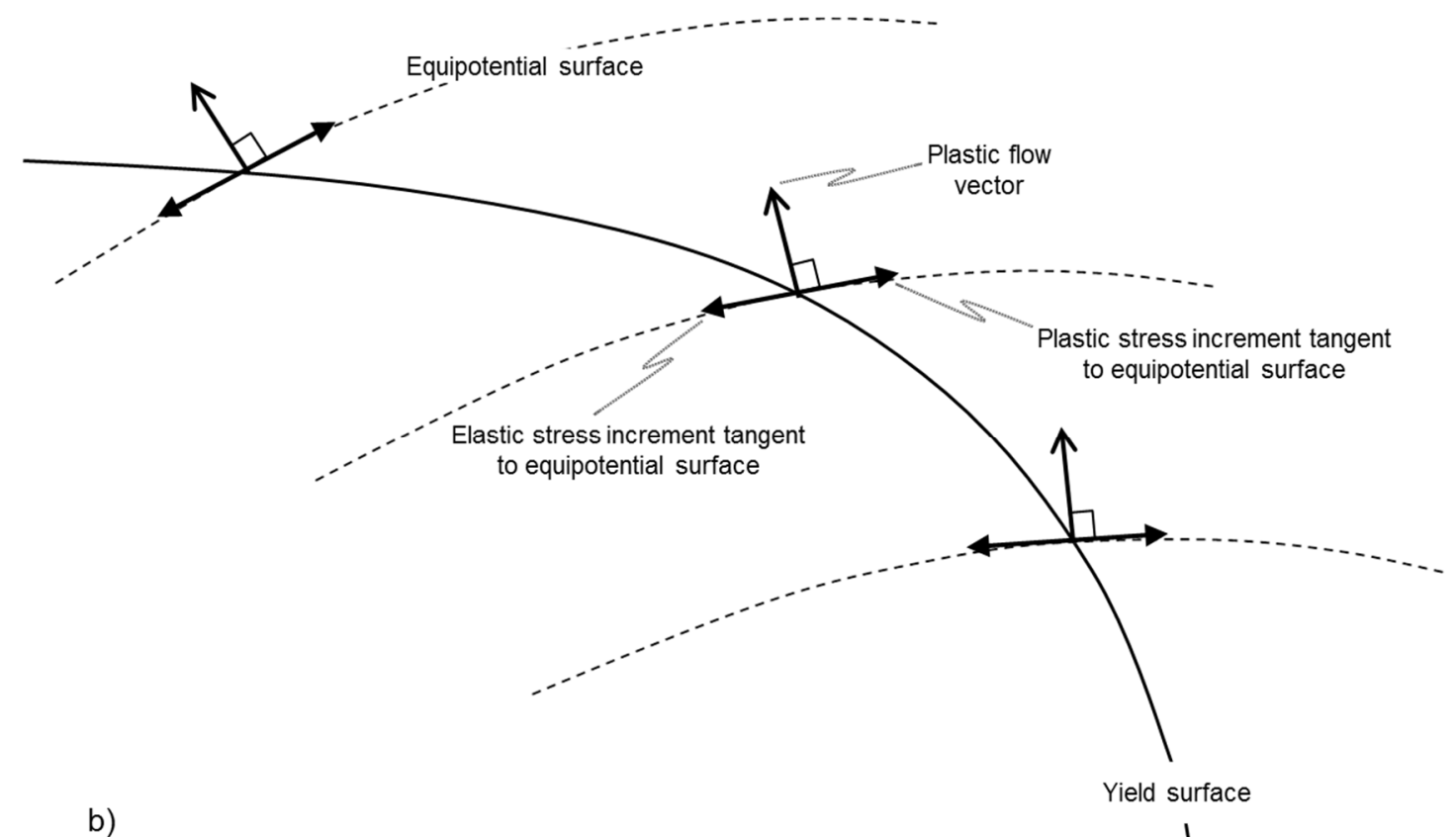

b)

\section{CAPTIONS}

Figure 1. Schematic representation of plastic yield and equipotential surfaces with a) associated flow rule and $b$ ) non-associated flow rule 